\title{
INVITED: Design Principles for True Random Number Generators for Security Applications
}

\author{
Miloš Grujić \\ COSIC, KU Leuven \\ Leuven, Belgium
}

\author{
Vladimir Rožić \\ COSIC, KU Leuven \\ Leuven, Belgium
}

\author{
David Johnston \\ Intel Corporation \\ Hilsboro, USA
}

\author{
John Kelsey \\ NIST \\ Gaithersburg, USA
}

\author{
Ingrid Verbauwhede \\ COSIC, KU Leuven \\ Leuven, Belgium
}

\begin{abstract}
The generation of high quality true random numbers is essential in security applications. For secure communication, we also require high quality true random number generators (TRNGs) in embedded and IoT devices. This paper provides insights into modern TRNG design principles and their evaluation, based on standard's requirements and design experience. We illustrate our approach with a case study of a recently proposed delay chain based TRNG.
\end{abstract}

ACM Reference Format:

Miloš Grujić, Vladimir Rožić, David Johnston, John Kelsey, and Ingrid Verbauwhede. 2019. INVITED: Design Principles for True Random Number Generators for Security Applications. In The 56th Annual Design Automation Conference 2019 (DAC '19), June 2-6, 2019, Las Vegas, NV, USA. ACM, New York, NY, USA, 3 pages. https://doi.org/10.1145/3316781.3323482

\section{INTRODUCTION}

True random number generator (TRNG) is an indispensable part of the security systems. Cryptographic algorithms and protocols base their security on the uniformity and the unpredictability (entropy) of the random numbers. Consequently, in the last 20 years, there has been a growing interest in TRNGs that generate random bits from intrinsically nondeterministic physical processes and satisfy high security requirements $[2,10]$.

Suitability of a TRNG for security applications cannot be merely evaluated by means of statistical tests (e.g., NIST SP 800-22 [12], FIPS 140-1 [9], DIEHARD [8]). These tests only assess the statistical quality of the output bits but not their unpredictability. Furthermore, significant statistical weaknesses of the noise source can be masked by the non-compressing post-processing algorithms that aim at improving statistics of the random numbers. Hence, modern TRNG design approach requires proper entropy justification and estimation [13] or stochastic model of the noise source [5]. Stochastic model is a formal probabilistic description of the physical phenomena that are used as a source of randomness, and whereby the lower bound on the entropy is estimated. This theoretical argument

Publication rights licensed to ACM. ACM acknowledges that this contribution was authored or co-authored by an employee, contractor or affiliate of the United States government. As such, the Government retains a nonexclusive, royalty-free right to publish or reproduce this article, or to allow others to do so, for Government purposes only.

DAC '19, June 2-6, 2019, Las Vegas, NV, USA

(C) 2019 Copyright held by the owner/author(s). Publication rights licensed to ACM. ACM ISBN 978-1-4503-6725-7/19/06 . \$ \$15.00

https://doi.org/10.1145/3316781.3323482

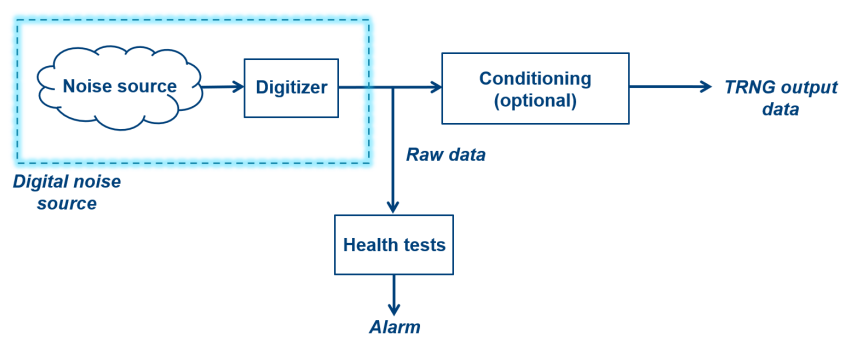

Figure 1: NIST SP 800-90B compliant TRNG architecture

for the TRNG unpredictability is a cornerstone of state-of-the-art TRNG design principles and the evaluation standards.

In this paper, we systematize the most relevant TRNG design principles for the high-end security systems. These principles should guide the system designer to develop a TRNG which can be reliably used as a root-of-trust in the security applications. We emphasize the significance of the security-by-design principle and compliance with the American [13] and the European standards [5].

The reminder of the paper is organized as follows. In Section 2, we present key TRNG concepts and NIST SP 800-90B recommendations on constructing entropy sources. In Section 3, we present challenges and approaches in generating random numbers on FPGAs. Finally, Section 4 summarizes main design principles and concludes the paper.

\section{ENTROPY SOURCES FOR CRYPTOGRAPHIC RANDOM NUMBER GENERATION}

NIST document SP 800-90B [13] provides recommendations for design and evaluation of the entropy sources for cryptographic applications. Entropy source (TRNG) is a nondeterministic component that produces random bits with a guaranteed minimum amount of entropy. SP 800-90B uses the notion of min-entropy, as it is the most conservative measure of the attacker's ability to guess the random value on the first try. The min-entropy is completely described by the probability of the most likely output of the entropy source. As illustrated in Figure 1, the entropy source is composed of a digital noise source, health tests and, optionally, conditioning.

The noise source is the core of the entropy source and it provides strings of raw random bits with a certain degree of the inherent 
unpredictability. Designer should provide justification for the nondeterministic behavior of the noise source and explanation why the noise source provides the claimed amount of entropy. Most commonly used noise sources are metastable flip flops, ring oscillators, noisy diodes, system interrupt timings and random memory initialization states.

Health tests are monitoring proper operation of the noise source and they are required for all entropy sources. These tests are examining raw random bits and are divided in three categories: startup, continuous and on-demand tests. Startup tests are executed once, after a power up and before any random bits are used. On the other hand, continuous tests are running during the entire entropy source operation and should quickly detect failures of the noise source. They do not require buffering of the random bits. On-demand tests are only run when requested and can be implemented as a rerun of the startup tests. If the designer does not create his own tests, SP $800-90 B$ provides two generic health tests that can be used: repetition count and adaptive proportion tests [13].

The role of the conditioning module is to improve statistics of the output bits by compressing streams of raw random bits into shorter bitstreams with increased entropy per bit. The designer of the entropy source can either choose his own conditioning function or he can use one of six vetted conditioning functions from SP 800-90B. Vetted conditioning functions are based on the wellunderstood cryptographic primitives and in certain cases they can claim full entropy of the output random bits. Conversely, non-vetted conditioning functions are never allowed to claim full entropy.

Evaluation of an entropy source in SP 800-90B implies estimation of entropy rate. This entropy rate is estimated by using set of statistical tests. The procedure starts with collecting adequate amount of data from continuous operation of the entropy source and data after resets of the entropy source. Afterwards, one of two tracks of tests is selected - IID (independent and identically distributed data) or non-IID track, depending on the nature of the noise source. The obtained initial entropy estimation and restart dataset are used as inputs to restart tests, which may lower the entropy estimate or completely fail the entropy source. If no major weaknesses are detected by the restart tests, the new entropy estimation for the entropy source is obtained. This value is the final entropy estimation if no conditioning is used. Otherwise, entropy estimation is updated once more, depending whether the vetted or non-vetted conditioning is applied. Non-vetted conditioning requires applying tests from IID or non-IID track once more, but on conditioned random numbers.

\section{TRUE RANDOM NUMBERS ON FPGAs}

Implementing TRNGs on FPGAs requires considerably different approach from the one used in ASIC designs, since FPGAs are developed to operate in a highly predictable way. In addition, the designer is constrained by the available resources that are already prefabricated on the chip. Implementation of FPGA-suitable TRNGs is further hindered by the design tools, which are not devised to cope with TRNG asynchronous blocks.

Available nondeterministic physical processes that can be used as noise sources on FPGAs are mostly restricted to the variable component of the logic delays, metastability of the flip-flops and

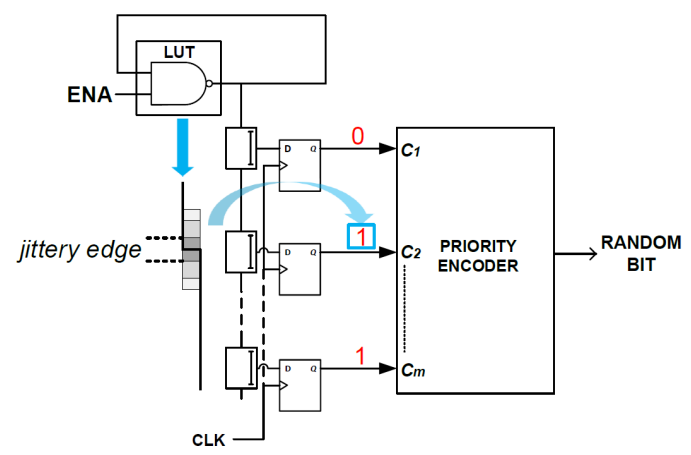

Figure 2: DC-TRNG architecture

initialization state of the memory elements. These processes must be rigorously analyzed and characterized in order to avoid overestimating the amount of generated entropy. Platform parameters that may affect the randomness of the output (e.g. LUT and routing delays) should be accounted in the stochastic model and measured prior to design. The measurements should be done by the on-chip circuitry, to avoid the signal filtering on the pad, package and probe.

Unfortunately, due to highly distinctive architectures between FPGAs of different vendors and generations, it is not always feasible to readily port TRNG designs between FPGAs. In cases where portability is achievable, the platform parameters processes might have rather different values implying that the security claim of the design has to be reevaluated for the target FPGA.

Many TRNG designs require additional effort of manual placement of the components and instantiations of low-level primitives $[10,11,16]$. This intervention generally needs to be done for each FPGA family but not for individual devices. On the other hand, certain designs $[1,6,10,14]$ require manual routing which often needs to be separately done for every individual device thereby increasing the design effort and lowering practical employment of the TRNG.

\subsection{Case Study: Delay Chain based TRNGs}

Two designs based on the delay chains have recently been proposed as feasible solutions for FPGA-suitable TRNGs that follow modern design principles: DC-TRNG [11] and ES-TRNG [16]. Both the DCTRNG and the ES-TRNG are highly portable, thanks to their fully digital architecture and availability of the delay chains in most commercial FPGAs $[4,16]$. The DC-TRNG is resistant to EM fault attacks [7] and can reliably operate across a broad temperature range. Additionally, it possesses stochastic model and achieves relatively high throughput with low design effort and stringent security guarantees. The ES-TRNG [16] is a low area version of the DC-TRNG with more intricate stochastic model.

The DC-TRNG uses timing jitter of the ring oscillator edge as a source of randomness, as depicted in Figure 2. Time position of jittery ring oscillator edge relative to the clock signal is captured in time-to-digital converter (TDC), which consists of a long delay chain and corresponding flip-flops. Subsequently, priority encoder translates the TDC position of the jittery edge into a raw random bit. Assessment of the jitter strength is performed by using the on-chip 
jitter measurement methodology presented in [15]. This method uses carry chains in a differential setup to remove undesirable influences of global and low frequency noises on the measurement of timing jitter. The original stochastic model of the DC-TRNG is based on the linearity assumption of the TDC. However, it was shown that TDCs on FPGAs are in practice far from linear and that this substantially impacts the claimed security level of the design. An enhanced stochastic model of the DC-TRNG [3] takes into account these non-linear effects and based on them reevaluates the amount of extracted entropy. The improved stochastic model of the DC-TRNG and the on-chip jitter measurement illustrate the importance of accurate measurement of all relevant physical parameters for the correct assessment of the security level. The DC-TRNG contains two online tests, designed following the TOTAL methodology [17]. This methodology is based on thorough testing of the raw bits while subjecting TRNG to various attacks and finding useful statistical features. The first online test detects attacks more efficiently, but with higher false positive rate. On the contrary, the second test is more robust, but it detects attacks less efficiently.

\section{CONCLUSION}

TRNGs are the root of security systems and therefore require well defined security models and conservative security guarantees. Based on the standard's requirements as well as industry and academia experience with designing TRNGs, we can identify four main design principles:

(1) Rely on theory. Stochastic models of physical noise sources are used to guarantee entropy of the raw random bits. In addition, theoretically secure post-processing algorithms such as entropy extractors are needed to ensure that these raw bits are transformed into full-entropy random numbers that are usable by applications.

(2) Check the assumptions. Models built on incorrect assumptions can lead to erroneous claims. Oversimplification of the model such as ignoring the effects of process variations, usually leads to overestimation of the generated entropy. The correct measurement of physical parameters is essential part of the model-building procedure.

(3) Check the results. Statistical tests are necessary at different stages of design validation and certification. While certainly not sufficient to prove security, these tests should still be performed as a sanity check.

(4) Test on-the-fly. Things can go wrong after deployment TRNG designs should be able to detect and respond to failures and attacks during the operation.

\section{ACKNOWLEDGMENTS}

This work was supported in part by the Research Council KU Leuven $\mathrm{C} 16 / 15 / 058$. In addition, this work was supported by the Flemish Government through FWO G.0876.14N, by the Hercules Foundation AKUL/11/19, and through the Horizon 2020 research and innovation programme under grant agreement Cathedral ERC Advanced Grant 695305. Vladimir Rožić is a Postdoctoral Fellow of the Fund for Scientific Research - Flanders (FWO).

\section{REFERENCES}

[1] Jean-Luc Danger, Sylvain Guilley, and Philippe Hoogvorst. 2009. High speed true random number generator based on open loop structures in FPGAs. Microelectronics journal 40, 11 (2009), 1650-1656.

[2] Viktor Fischer. 2012. A Closer Look at Security in Random Number Generators Design. In Constructive Side-Channel Analysis and Secure Design (Lecture Notes in Computer Science), Werner Schindler and Sorin A. Huss (Eds.). Springer Berlin Heidelberg, 167-182.

[3] Miloš Grujić, Vladimir Rožić, Bohan Yang, and Ingrid Verbauwhede. 2018. A Closer Look at the Delay-Chain based TRNG. In 2018 IEEE International Symposium on Circuits and Systems (ISCAS). 1-5.

[4] Miloš Grujić, Bohan Yang, Vladimir Rožić, and Ingrid Verbauwhede. 2018. Towards inter-vendor compatibility of true random number generators for FPGAs. In 2018 Design, Automation Test in Europe Conference Exhibition (DATE). 15201523.

[5] Wolfgang Killmann and Werner Schindler. 2011. A proposal for: Functionality classes for random number generators. ser. BDI, Bonn (2011).

[6] Florent Lozach, Molka Ben-Romdhane, Tarik Graba, and Jean-Luc Danger. 2013. FPGA Design of an Open-Loop True Random Number Generator. In $2013 \mathrm{Eu}$ romicro Conference on Digital System Design. 615-622.

[7] Maxime Madau, Michel Agoyan, Josep Balasch, Miloš Grujić, Patrick Haddad, Philippe Maurine, Vladimir Rožić, Dave Singelée, Bohan Yang, and Ingrid Verbauwhede. 2018. The Impact of Pulsed Electromagnetic Fault Injection on True Random Number Generators. In 2018 Workshop on Fault Diagnosis and Tolerance in Cryptography (FDTC). 43-48.

[8] George Marsaglia. 1995. DIEHARD Test Suite. (1995). http://www.stat.fsu.edu/ pub/diehard/

[9] NIST. 1994. FIPS 140-1: Security Requirements for Cryptographic Modules. Technical Report. https://csrc.nist.gov/publications/detail/fips/140/1/archive/1994-01-11

[10] Oto Petura, Ugo Mureddu, Nathalie Bochard, Viktor Fischer, and Lilian Bossuet. 2016. A survey of AIS-20/31 compliant TRNG cores suitable for FPGA devices. In Field Programmable Logic and Applications (FPL), 2016 26th International Conference on. IEEE, 1-10.

[11] Vladimir Rožić, Bohan Yang, Wim Dehaene, and Ingrid Verbauwhede. 2015. Highly efficient entropy extraction for true random number generators on FPGAs. In Design Automation Conference (DAC), 2015 52nd ACM/EDAC/IEEE. IEEE, 1-6.

[12] Andrew L. Rukhin, Juan Soto, James R. Nechvatal, Miles E. Smid, Stefan D. Leigh, M. Levenson, M. Vangel, Nathanael A. Heckert, and D. L. Banks. 2010. A Statistical Test Suite for Random and Pseudorandom Number Generators for Cryptographic Applications. Special Publication (NIST SP) - 800-22 Rev 1a (Sept. 2010).

[13] Meltem Sönmez Turan, Elaine Barker, John Kelsey, Kerry McKay, Mary Baish, and Mike Boyle. 2018. Recommendation for the entropy sources used for random bit generation. NIST Special Publication 800-90B (Jan. 2018), 84.

[14] Michal Varchola and Miloš Drutarovský. 2010. New High Entropy Element for FPGA Based True Random Number Generators. In Cryptographic Hardware and Embedded Systems, CHES 2010 (Lecture Notes in Computer Science), Stefan Mangard and François-Xavier Standaert (Eds.). Springer Berlin Heidelberg, 351-365.

[15] Bohan Yang, Vladimir Rožić, Miloš Grujić, Nele Mentens, and Ingrid Verbauwhede. 2017. On-chip jitter measurement for true random number generators. In 2017 Asian Hardware Oriented Security and Trust Symposium (AsianHOST). IEEE, 9196.

[16] Bohan Yang, Vladimir Rožić, Miloš Grujić, Nele Mentens, and Ingrid Verbauwhede. 2018. ES-TRNG: A High-throughput, Low-area True Random Number Generator based on Edge Sampling. IACR Transactions on Cryptographic Hardware and Embedded Systems 2018, 3 (Aug. 2018), 267-292.

[17] Bohan Yang, Vladimir Rožić, Nele Mentens, Wim Dehaene, and Ingrid Verbauwhede. 2016. TOTAL: TRNG on-the-fly testing for attack detection using Lightweight hardware. In 2016 Design, Automation Test in Europe Conference Exhibition (DATE). 127-132. 